\title{
Ultimate Support Force of Excavation Face in Curved Shield Tunnels in Composite Strata
}

\author{
Chunquan DAI, Quanlei WANG*, Kun JIANG, Tao ZHENG
}

\begin{abstract}
For curved shield tunnels in composite strata, the excavation face may undergo sliding failure due to the interface effect of soil layers, the over-excavation of shield cutterhead, and the asymmetrical thrust of jacks. Inspired by the silo theory, this paper establishes the calculation model and formula for the ultimate support pressure in the excavation face of irregular curved trapezoidal prism in composite strata, and analyses the variation law of the excavation face at different soil layer thickness ratios and curvature radii through the case study. The results show that the horizontal displacement of the excavation face in curved shield tunnel in composite strata changes suddenly at the interface between hard and soft soil layers; under the eccentric support force, the failure of the excavation face is asymmetrically distributed, and the ultimate support pressure increases first and then decreases with the decline of the curvature radius; the support pressure is biased towards the outside of the curve and greater than that of straight shield tunnel in single stratum; the ultimate support pressure increased with the soil cohesion and internal friction angle of the excavation face, and increased first and then declined with the growth of the rupture angle difference between the left and right sides of the soil mass before the excavation face; when the rupture angle difference exceeds 1.28 , the ultimate support pressure gradually stabilizes.
\end{abstract}

Keywords: curved shield tunnels in composite strata; equilibrium model for irregular curved trapezoidal soil mass; excavation face destabilization; silo theory; ultimate support pressure

\section{INTRODUCTION}

Rapid urbanization has induced a continued growth of the travel demand among urban residents. To satisfy the rising demand, rail transit has become the dominant trend of traffic development thanks to its safety, comfort, fast speed, high transport volume, and environmental friendliness. Nevertheless, the excavation face may lose stability during the construction of rail transit system, leading to severe damages. Similar engineering disasters (e.g. instability of excavation face, displacement of shield, and large deformation of the ground) are extremely likely to occur during the construction of curved shield tunnels in composite strata, owing to the complex stress distribution on the excavation face [1-3], if the support pressure on the excavation face is not adjusted in a timely manner. To control the stability of excavation face and other engineering disasters, it is of great theoretical and practical significance to investigate the deformation law of excavation face and ultimate support pressure of curved shield tunnels in composite strata.

The existing studies on the ultimate support pressure of excavation face mainly adopt the ultimate equilibrium method [4-10] and the ultimate analysis method [11, 12]. Most of these studies concentrate on straight shield tunnels in single stratum. There is no report on the excavation face stability of curved shield tunnels in composite strata. Inspired by the silo theory, this paper establishes an ultimate equilibrium model for curved shield tunnels in composite strata and derives the calculation formula for the support pressure of the excavation face. Focusing on a small curvature shield tunnel in variable strata of Jinan Metro Line R1, the proposed model and formula were coupled with numerical simulation to determine the failure mode of excavation face, damages on surrounding rock and the ultimate support pressure of curved shield tunnels with different radii in composite strata under the unbalanced thrust of jacks, and to disclose the impacts of stratum thickness ratio, rupture angle difference, cohesion and internal friction angle on the stability of the excavation face.

\section{ULTIMATE SUPPORT PRESSURE OF EXCAVATION FACE}

\subsection{Ultimate Equilibrium Model}

The most popular stability analysis model of shield tunnel excavation face is the wedge-shaped prism model based on the silo theory. Nevertheless, the excavation face of curved shield tunnels in composite strata is under the unbalanced thrust of jacks on the two sides of the geological interface. In this case, an interface will appear in the soil mass before the excavation face, and the two sides of the excavation face will be subjected to asymmetric force. In view of these, this paper proposes an ultimate equilibrium model for irregular curved trapezoid (Fig. 1).

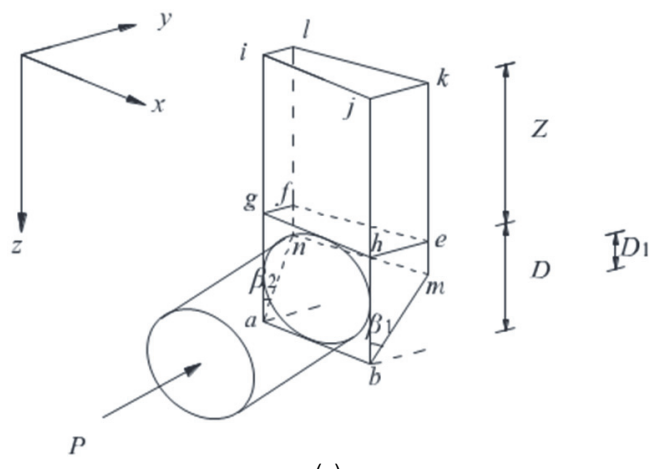

(a)

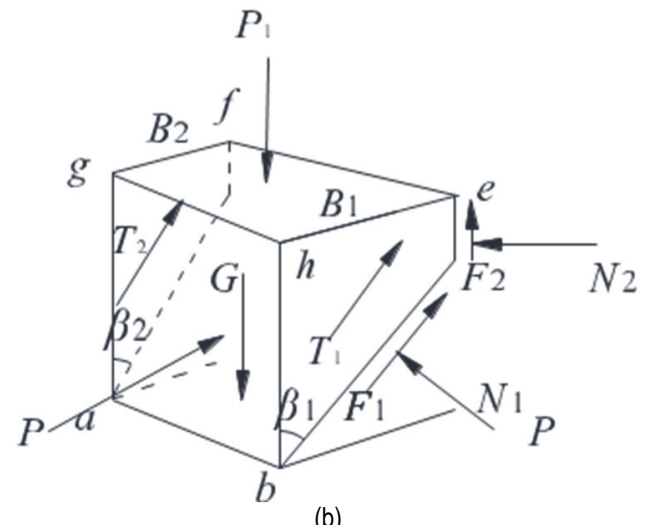

Figure 1 Ultimate equilibrium model for irregular curved trapezoid 
In Fig. 1a, abhg is the excavation face of the tunnel; anfg-bmeh is the sliding irregular curved trapezoidal soil mass when the excavation face becomes unstable; ghef-ijkl is the sliding right-angled trapezoidal soil mass; anfg, bmeh, abmn and mefn are sliding surfaces; ijkl is the ground surface; $\beta_{1}$ and $\beta_{2}$ are the rupture angles of the sliding soil mass in the excavation face; $D$ is the tunnel diameter; $P$ is the total support pressure from the shield cutter on the excavation face.

In Fig. $1 \mathrm{~b}, B_{1}$ and $B_{2}$ are the bottom lengths of the trapezoidal soil mass; $P_{1}$ is the force from the trapezoidal soil mass acting on the lower irregular trapezoid; $G$ is the self-weight of the irregular trapezoidal soil mass; $F_{1}, F_{2}, T_{1}$ and $T_{2}$ are respectively the total shear forces on the corresponding surfaces; $N_{1}$ and $N_{2}$ are respectively the total pressures acting on the corresponding surfaces.

The ultimate equilibrium model for irregular curved trapezoid was established and implemented under the following hypotheses:

(1) The soil mass is isotropic, and the sliding surface soil conforms to the Mohr-Coulomb failure criterion. The shear strength $\tau$ of the soil can be expressed as:

$\tau=c+\tan \varphi$

where $c$ is the soil cohesion, the unit is $\mathrm{kPa} ; \sigma$ is the normal stress on the shear plane; $\varphi$ is the friction angle in the soil mass.

(2) The area damaged by the excavation face consists of the lower irregular trapezoidal soil mass and the upper trapezoidal soil mass.

(3) The contact surface between the irregular curved trapezoidal soil mass and the excavation face is a square whose side length equals the tunnel diameter $D$.

(4) The stress is evenly distributed on the top surface and inclined sliding surface of the irregular curved trapezoidal soil mass.

(5) Similar to that on the vertical surface, the vertical stress on the inclined surface of the irregular curved trapezoidal soil mass increases linearly with depth.

(6) The impact of underground seepage on the stability of the shield tunnel excavation face is not considered.

\subsection{Calculation Formula of Ultimate Support Pressure on Excavation Face}

According to the force diagram of the irregular curved trapezoidal soil mass in Fig. 1b, the equilibrium between vertical and horizontal forces on the trapezoidal soil mass in composite strata can be expressed as follows when the soil before the excavation face is in the ultimate equilibrium state:

$$
\begin{aligned}
& P_{1}+G=T_{1} \cos \beta_{1}+T_{2} \cos \beta_{2}+F_{1} \int_{\beta_{2}}^{\beta_{1}} \cos \theta \mathrm{d} \theta+F_{2}= \\
& =N_{1} \int_{\beta_{2}}^{\beta_{1}} \sin \theta \mathrm{d} \theta \\
& P+T_{1} \sin \beta_{1}+T_{2} \sin \beta_{2}+F_{1} \int_{\beta_{2}}^{\beta_{1}} \sin \theta \mathrm{d} \theta= \\
& =N_{1} \int_{\beta_{2}}^{\beta_{1}} \cos \theta \mathrm{d} \theta+N_{2}
\end{aligned}
$$

Integrating Eqs. (2) and (3), the result can be simplified as:

$$
\begin{aligned}
& P=\frac{P_{1}+G-T_{1} \cos \beta_{1}-T_{2} \cos \beta_{2}-F_{1}\left(\sin \beta_{2}-\sin \beta_{1}\right)-F_{2}}{\cos \beta_{1}-\cos \beta_{2}} . \\
& \cdot\left(\sin \beta_{1}-\sin \beta_{2}\right)-T_{1} \sin \beta_{1}-T_{2} \sin \beta_{2}- \\
& -F_{1}\left(\cos \beta_{2}-\cos \beta_{1}\right)+N_{2}
\end{aligned}
$$

The unknown parameters in Eq. (4) can be calculated as follows.

1) The force from the trapezoidal soil mass on the irregular wedge $\left(P_{\mathrm{v} 0}\right)$

$$
P_{1}=\sigma_{\mathrm{v}} S_{e f g h}=\sigma_{\mathrm{v}}\left(B_{1}+B_{2}\right) \cdot \frac{D}{2}
$$

where $\sigma_{\mathrm{v}}$ is the vertical stress from the trapezoidal soil mass acting on the irregular curved trapezoidal soil mass; $S_{\text {efgh }}$ is the area of the efgh plane.

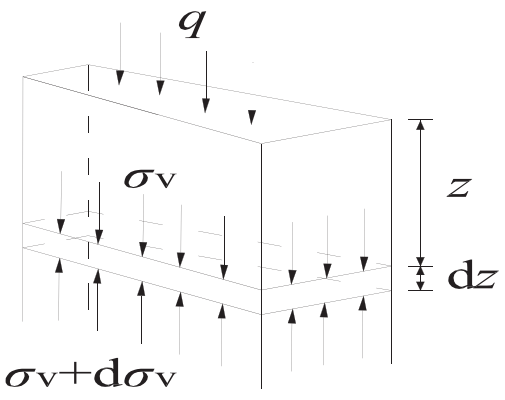

(a) 3D loose earth pressure model

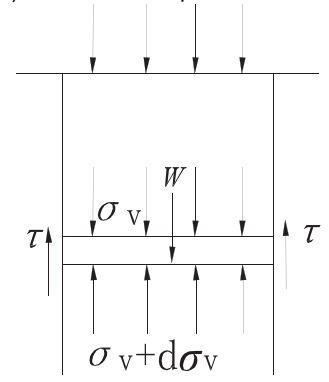

(b) Vertical force model of soil micro-element Figure 2 Calculation model of loose earth pressure

According to Terzaghi's theory on loose earth pressure [13], the 3D compressive stress model of the soil mass and the vertical force model of soil micro-element at depth $z$ were constructed (Fig. 2). Note that $q$ is the ground overload; $w$ is the self-weight of soil micro-element; $\tau$ is the shear stress on the side of soil micro-element.

As shown in Fig. 2b, the vertical force equilibrium equation of the soil micro-element can be established as:

$$
\begin{aligned}
& \sigma_{\mathrm{V}} S_{e f g h}+\gamma S_{e f g h} \mathrm{~d} z=\left(\sigma_{\mathrm{v}}+\mathrm{d} \sigma_{\mathrm{v}}\right) S_{e f g h}+\tau B_{1} \mathrm{~d} z+ \\
& +\tau B_{2} \mathrm{dz}+\tau D \mathrm{~d} z+\tau\left[D^{2}+\left(B_{1}-B_{2}\right)^{2}\right]^{\frac{1}{2}} \mathrm{~d} z
\end{aligned}
$$

where $\tau=c+K \sigma_{\mathrm{v}} \tan \varphi ; K$ is the lateral pressure coefficient of the trapezoidal soil mass.

The integral from of Eq. (6) can be expressed as: 


$$
\begin{aligned}
& \frac{\mathrm{d} \sigma_{\mathrm{v}}}{\mathrm{d} z}+\frac{K \tan \varphi}{S_{\text {efgh }}}\left\{B_{1}+B_{2}+D+\left[D^{2}+\left(B_{1}-B_{2}\right)^{2}\right]^{\frac{1}{2}}\right\} \sigma_{\mathrm{v}} \\
& =\gamma-\frac{c}{S_{\text {efgh }}}\left\{B_{1}+B_{2}+D+\left[D^{2}+\left(B_{1}-B_{2}\right)^{2}\right]^{\frac{1}{2}}\right\}
\end{aligned}
$$

Eq. (7) is a first-order non-homogeneous linear differential equation. Solving this equation, we have:

$\sigma_{\mathrm{v}}=\mathrm{A}_{0} \mathrm{e}^{-P_{0} z}+\frac{Q_{0}}{P_{0}}$

where $\mathrm{A}_{0}$ is a constant to be determined:

$$
\begin{aligned}
& P_{0}=\frac{K \tan \varphi}{S_{\text {efgh }}}\left\{B_{1}+B_{2}+D+\left[D^{2}+\left(B_{1}-B_{2}\right)^{2}\right]^{\frac{1}{2}}\right\} \\
& Q_{0}=\gamma-\frac{c}{S_{\text {efgh }}}\left\{B_{1}+B_{2}+D+\left[D^{2}+\left(B_{1}-B_{2}\right)^{2}\right]^{\frac{1}{2}}\right\}
\end{aligned}
$$

According to the boundary conditions $\left.\sigma_{\mathrm{v}}\right|_{z=0}=q$, we have:

$$
A_{0}=q-\frac{Q_{0}}{P_{0}}
$$

Substituting Eq. (10) into Eq. (8), we have:

$$
\sigma_{\mathrm{v}}=\frac{Q_{0}}{P_{0}}\left(1-\mathrm{e}^{-P_{0} z}\right)+q \mathrm{e}^{-P_{0} z}
$$

2) Self-weight of irregular curved trapezoidal soil mass $(G)$

Since the inclined surface of the irregular trapezoidal soil mass is a curved surface, the wedge was meshed into grids in the $x$ direction for gravity calculation (Fig. 3).

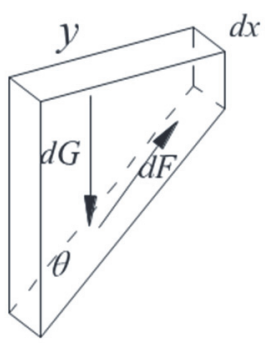

Figure 3 Trapezoidal micro-element in the $x$ direction

$$
\begin{aligned}
& G=\gamma V=\gamma \int_{0}^{D} d V=\gamma \int_{0}^{D} \frac{1}{2}(2 D-y \cot \beta) y \mathrm{~d} x= \\
& =\frac{1}{2} \int_{\beta_{2}}^{\beta_{1}} \int_{0}^{D}\left\{2 D-\left[\frac{\left(B_{1}-B_{2}\right)}{D} x+B_{2}\right] \cot \theta\right\}\left[\frac{\left(B_{1}-B_{2}\right)}{D} x+B_{2}\right] \mathrm{d} x \mathrm{~d} \theta= \\
& =\left(\frac{B_{1}-3 B_{2}}{2}\right) D^{2}-D \ln \frac{\sin \beta_{1}}{\sin \beta_{2}}\left[\frac{\left(B_{1}-B_{2}\right)^{2}}{6}+\frac{B_{2}{ }^{2}}{2}\right]
\end{aligned}
$$

where $\gamma$ is the saturated specific weight of the soil, the unit is $\mathrm{kN} / \mathrm{m}^{3} ; V$ is the volume of the irregular trapezoidal soil mass.
3) Shear force on the inclined surface of the irregular trapezoidal soil mass $\left(F_{1}\right)$

According to the grids of the irregular curved trapezoidal soil mass (Fig. 3) and the hypothesis that the vertical stress increases linearly with depth, the total shear force on the inclined surface $\mathrm{F}$ can be expressed as:

$$
F_{1}=\int_{0}^{D} \mathrm{~d} x \int_{\beta_{2}}^{\beta_{1}} \frac{1}{\sin \theta} \mathrm{d} \theta \int_{B_{2} \sin \beta_{2}}^{B_{1} \sin \beta_{1}} y \mathrm{~d} y\left(c+N_{1} \tan \varphi\right)
$$

Substituting Eq. (2) into Eq. (13), we have:

$$
\begin{aligned}
& F_{1}=\frac{D}{2} \frac{\cos \beta_{1}-\cos \beta_{2}}{\cos \beta_{1}-\cos \beta_{2}+\sin \beta_{2}-\sin \beta_{1}} . \\
& \cdot \ln \frac{\csc \beta_{1}-\cot \beta_{1}}{\csc \beta_{2}-\cot \beta_{2}}\left(B_{1}{ }^{2} \sin ^{2} \beta_{1}-B_{2}{ }^{2} \sin ^{2} \beta_{2}\right) . \\
& \cdot\left(c+\frac{P_{1}+G-T_{1} \cos \beta_{1}-T_{2} \cos \beta_{2}-F_{2}}{\cos \beta_{1}-\cos \beta_{2}} \tan \varphi\right)
\end{aligned}
$$

4) Shear forces on the vertical sliding surfaces bmeh and $\operatorname{anfg}\left(T_{1}\right.$ and $\left.T_{2}\right)$

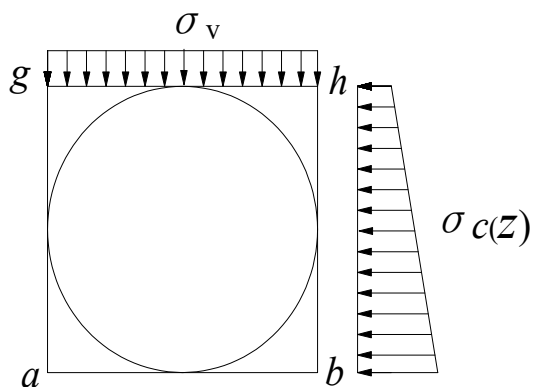

(a) Vertical surface stress distribution

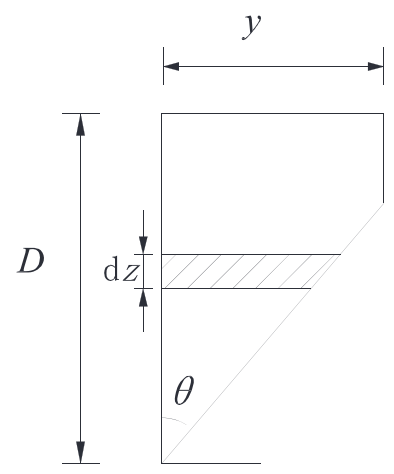

(b) Vertical surface area integral Figure 4 Calculation diagram of shear force $T$

The shear forces on the vertical sliding surfaces can be calculated against Fig. 4. Let $\sigma_{\mathrm{v}}$ be the vertical stress on the top surface ghef of the irregular curved trapezoidal soil mass. According to the hypothesis that the vertical stress increases linearly with depth, the vertical stress $\sigma_{\mathrm{v}}(z)$ at each point on the two sides of the sliding surface can be expressed as:

$$
\sigma_{\mathrm{v}}(z)=\sigma_{\mathrm{v}}+\gamma z
$$

From the traditional silo theory, the lateral pressure $\sigma_{\mathrm{v}}(c)$ at each point on the two sides of the sliding surface can be obtained as: 
$\sigma_{\mathrm{c}}(z)=K \sigma_{\mathrm{v}}(z)$

where $K(K=1-\sin \varphi)$ is the lateral pressure coefficient of the soil mass [14].

Taking the micro-elements on surfaces bmeh and $a n f g$ (Fig. 4b), the shear force acting on the area of each microelement can be expressed as:

$\mathrm{d} T=\left[c+\sigma_{c}(z) \tan \varphi\right] \mathrm{d} s$

Integrating Eq. (17), we have the shear forces $T_{1}$ and $T_{2}$ on each side of the sliding surface:

$$
\begin{aligned}
& T_{1}=\int_{0}^{B_{1}} \mathrm{~d} T_{1}=\int_{0}^{D-B_{1} \cot \beta_{1}}\left[c+\sigma_{\mathrm{c}}(z) \tan \varphi\right] B_{1} \mathrm{~d} z+ \\
& +\int_{D-B_{1} \cot \beta_{1}}^{D}\left[c+\sigma_{c}(z) \tan \varphi\right](D-z) \tan \beta_{1} \mathrm{~d} z= \\
& =c D B_{1}-\frac{c\left(2 D B_{1}-B_{1}^{2} \cot \beta_{1}\right)}{2}+K D \sigma_{\mathrm{v}} B_{1} \tan \varphi- \\
& -\frac{K \gamma}{3}\left(3 D^{2} B_{1}+3 D B_{1}{ }^{2} \cot \beta_{1}+B_{1}{ }^{3} \cot ^{2} \beta_{1}\right) \tan \varphi \\
& T_{2}=\int_{0}^{B_{2}} \mathrm{~d} T_{2}=\int_{0}^{D-B_{2} \cot \beta_{2}}\left[c+\sigma_{\mathrm{c}}(z) \tan \varphi\right] B_{2} \mathrm{~d} z+ \\
& +\int_{D-B_{2} \cot \beta_{2}}^{D}\left[c+\sigma_{c}(z) \tan \varphi\right](D-z) \tan \beta_{2} \mathrm{~d} z= \\
& =c D B_{2}-\frac{c\left(2 D B_{2}-B_{2}{ }^{2} \cot \beta_{2}\right)}{2}+K D \sigma_{\mathrm{v}} B_{2} \tan \varphi- \\
& -\frac{K \gamma}{3}\left(3 D^{2} B_{2}+3 D B_{2}{ }^{2} \cot \beta_{2}+B_{2}{ }^{3} \cot ^{2} \beta_{2}\right) \tan \varphi
\end{aligned}
$$

Through integral operation, we have:

5) The shear force $\left(F_{2}\right)$ and pressure $\left(N_{2}\right)$ on the vertical surface mefn of the irregular curved trapezoidal soil mass.

The stress distribution on the vertical surface mefn and its calculation process are similar to those of shear forces $T_{1}$ and $T_{2}$ :

$$
F_{2}=\left(D-\frac{B_{1}}{\tan \beta_{1}}\right) \sqrt{D^{2}+\left(B_{1}-B_{2}\right)^{2}}\left[c+\sigma_{\mathrm{c}}(z) \tan \varphi\right](20)
$$

For the pressure $N_{2}$ can be derived from the earth pressure calculation formula:

$$
N_{2}=\left(D-\frac{B_{1}}{\tan \beta_{1}}\right) \sqrt{D^{2}+\left(B_{1}-B_{2}\right)^{2}}\left[\frac{\gamma}{2}\left(D-\frac{B_{1}}{\tan \beta_{1}}\right)^{2} K\right]
$$

Substituting Eqs. (5), (9), (11), (12), (14), (18), (19), (20) and (21) into Eq. (4), we have the calculation formula of the ultimate support pressure $P$ on the excavation face of small curvature curved shield tunnels in composite strata:

$$
\begin{aligned}
& P=\frac{3 D \sigma_{\mathrm{v}}\left(B_{1}+B_{2}\right)+3 D^{2}\left(B_{1}-3 B_{2}\right)}{6\left(\cos \beta_{1}-\cos \beta_{2}\right)}- \\
& -\frac{3 D \ln \frac{\sin \beta_{1}}{\sin \beta_{2}}\left[\left(B_{1}-B_{2}\right)^{2}+3 B_{2}^{2}\right]}{6\left(\cos \beta_{1}-\cos \beta_{2}\right)}-\frac{T_{1} \cos \beta_{1}-T_{2} \cos \beta_{2}}{\cos \beta_{1}-\cos \beta_{2}}- \\
& -F_{1} \delta-\eta\left\{-\delta \frac{\left[c+\sigma_{\mathrm{c}}(z) \tan \varphi\right]}{\cos \beta_{1}-\cos \beta_{2}}-\frac{\gamma}{2}\left(D-\frac{B_{1}}{\tan \beta_{1}}\right)^{2} K\right\}- \\
& -F_{1} \delta-\eta\left\{-\delta \frac{\left[c+\sigma_{\mathrm{c}}(z) \tan \varphi\right]}{\cos \beta_{1}-\cos \beta_{2}}-\frac{\gamma}{2}\left(D-\frac{B_{1}}{\tan \beta_{1}}\right)^{2} K\right\} \\
& \delta=\frac{\sin \beta_{1}-\sin \beta_{2}}{\cos \beta_{2}-\cos \beta_{1}} \\
& \eta=\left(D-\frac{B_{1}}{\tan \beta_{1}}\right) \sqrt{D^{2}+\left(B_{1}-B_{1}\right)^{2}}
\end{aligned}
$$

\section{CASE STUDY}

This section aims to verify the feasibility of the proposed calculation model on the ultimate support pressure of curved shield tunnels in composite strata, which is based on the silo theory. For this purpose, the proposed model was applied to a shield tunnel in composite strata of Jinan Metro Line $R_{1}$.

\subsection{Overview}

\begin{tabular}{|c|c|c|c|c|c|c|}
\hline Name & $\begin{array}{l}\text { Thickness } \\
\text { / m }\end{array}$ & $\begin{array}{c}\text { Bulk weight } \\
/ \mathrm{kN} \cdot \mathrm{m}^{-3}\end{array}$ & $\begin{array}{c}\text { Elastic modulus } \\
/ \mathrm{MPa}\end{array}$ & Poisson's ratio & $\begin{array}{l}\text { Cohesion } \\
/ \mathrm{kPa}\end{array}$ & $\begin{array}{c}\text { Internal friction } \\
\text { angle } /{ }^{\circ}\end{array}$ \\
\hline Miscellaneous fill & 5.9 & 17.5 & 8 & 0.35 & 10 & 16 \\
\hline Loess & 4.1 & 18.5 & 12 & 0.32 & 20 & 18 \\
\hline Silty clay & 9.8 & 19.0 & 30 & 0.3 & 24 & 26 \\
\hline Clay & 12.7 & 18.0 & 40 & 0.28 & 30 & 34 \\
\hline Cemented sand and pebbles & 7.3 & 21.0 & 80 & 0.26 & 40 & 24 \\
\hline Clay & 7.4 & 19.0 & 50 & 0.28 & 32 & 35 \\
\hline Moderately weathered limestone & 12.8 & 22.0 & 200 & 0.24 & 100 & 28 \\
\hline Shield segment & 0.30 & 25.0 & 34500 & 0.20 & & \\
\hline Grout & 0.25 & 23.0 & 50 & 0.22 & & \\
\hline
\end{tabular}

The target section of Jinan Metro Line $R_{1}$ was excavated by curved shield with a radius $R=300 \mathrm{~m}$, using an earth pressure balance shield machine. The total length of the tunnel is $l=9.4 \mathrm{~m}$, the outer and inner diameters of the lining ring are $6.4 \mathrm{~m}$ and $5.8 \mathrm{~m}$, respectively, and the width and thickness of the shield segment are $1.2 \mathrm{~m}$ and 0.3 $\mathrm{m}$, respectively. The project is located in a thick alluvial plain. From top to bottom, the strata consist of miscellaneous fill, loess, silty clay, clay, cemented sand and pebbles, clay, and moderately weathered limestone [7]. The tunnel body lies within the composite strata of clay and moderately weathered limestone. The distribution and physical-mechanical parameters of each stratum are listed in Tab. 1.

Table 1 Physical-mechanical parameters of each stratum 


\subsection{Thrust Analysis of Excavation Face of Curved Shield Tunnel}

During the excavation of the curved shield tunnel in composite strata, the shield direction is adjusted by setting the thrusts $\left(q_{1}, q_{2}\right.$ and $\left.q_{3}\right)$ and strokes of different groups of shield tail jack. According to the design data, the jack thrusts were divided into four groups (Fig. 5) with the angle of $67.5^{\circ}, 90^{\circ}, 112.5^{\circ}$ and $90^{\circ}$, respectively. The shield is turned to the left when the Group A thrust equals Group C thrust, and Group B thrust surpasses Group D thrust.

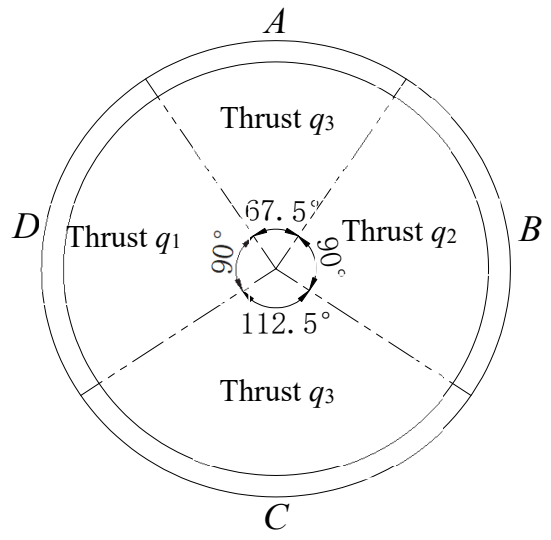

Figure 5 Trust of each group of jacks

\subsection{Calculation Results and Analysis}

(1) Influence of the rupture angle $\beta$ of the slider

The ultimate support force of the excavation face of the curved shield tunnel depends on the rupture angle $\beta$. In the soil mass before the excavation face, the Group B thrust is greater than the Group $\mathrm{D}$ thrust, i.e., $\beta_{1}>\beta_{2}$. The support force of the excavation face hinges on both $\beta_{1}$ and $\beta_{2}$. To determine the ultimate support force of the excavation face at different values of $\beta$, the trial iteration method was employed for calculation [15-20].

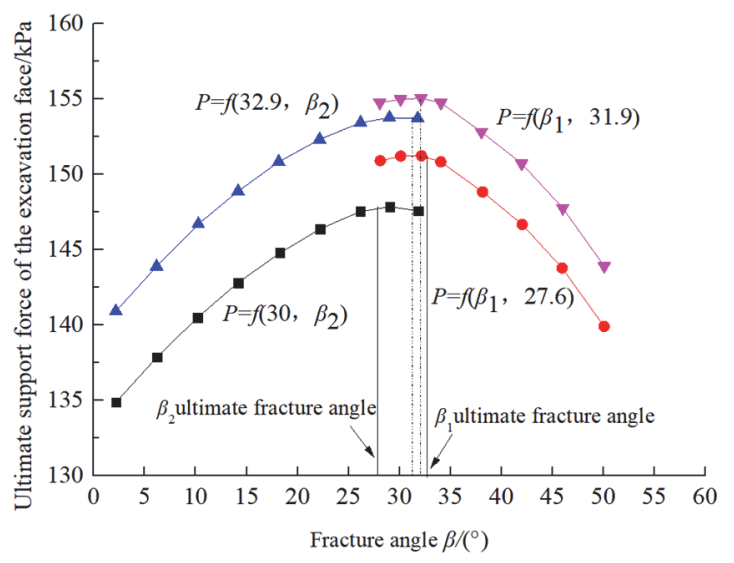

Figure 6 Relationship between ultimate support force and fracture angle

According to the physical-mechanical parameters of the strata, a computer program was developed to obtain the relationship (the lower left curve in Fig. 6) between the ultimate support force $P$ and $\beta_{2}$ when the rupture angle of the slider $\beta_{1}=45^{\circ}-\frac{\varphi}{2}=30^{\circ}[6]$. It can be seen that the rupture angle $\beta_{2}=27.6^{\circ}$ when the excavation surface reached the ultimate support force. Then, the relationship (the lower right curve in Fig. 6) between $P$ and $\beta_{1}$ was derived at the rupture angle of $\beta_{2}=27.6^{\circ}$. It can be seen that the rupture angle $\beta_{1}=32.9^{\circ}$ when the excavation surface reached the ultimate support force. The accuracy is $[(32.9-30) / 30] \cdot 100 \%=9.7 \%$.

Based on the rupture angle $\beta_{1}=32.9^{\circ}$ obtained in the first iteration, the second iteration was carried out to determine the value of $\beta_{2}\left(31.9^{\circ}\right)$ at the maximum support force (the upper left curve in Fig. 6). Assuming that $\beta_{2}=$ $31.9^{\circ}$, the author derived the relationship (the upper right curve in Fig. 6) between the ultimate support force $P$ and $\beta_{1}$. It can be seen that the rupture angle $\beta_{1}=32.7^{\circ}$ when the excavation surface reached the ultimate support force. The accuracy is $[(32.9-32.7) / 32.9] \cdot 100 \%=0.6 \%$. According to some engineering problems and numerical analysis methods [20], the solution is usually deemed as accurate when the error falls within 3\%. Therefore, the termination condition of our iterations was set as the error $<3 \%$.

Then, a similar iteration process was performed at the rupture angle $\beta_{2}=45^{\circ}-\frac{\varphi}{2}=30^{\circ}$ [6]. The result is not discussed in details, as it is similar to the previous rupture angle. Therefore, the support force reached the maximum at $\beta_{1}=32.7^{\circ}$ and $\beta_{2}=31.9^{\circ}$. In addition, Fig. 6 shows that the ultimate support force increased first and then decreased with the increase of the rupture angle; the support force of the excavation face was relatively small when there was a large difference between the rupture angles on the two sides of the excavation face.

(2) Influence of curvature radius $R$ on the rupture angle $\beta$

As shown in Fig. 7, the front and back sides of the trapezoidal soil mass on the excavation face are extended towards the inside of the curve. Let the length of each extension line be $\mathrm{R}$, the radius of the small curvature shield tunnel. According to geometric relationship, we have $\tan \beta_{2}=R \tan \beta_{1} /(R+D)$. Substituting the curvature radius $R=300 \mathrm{~m}$ into this formula, we have $\beta_{2}=32.1^{\circ}$ when $\beta_{1}=32.7^{\circ}$. The value is not much different from the fracture angle $\beta 2$ obtained from the previous analysis, thus verifying this hypothesis.

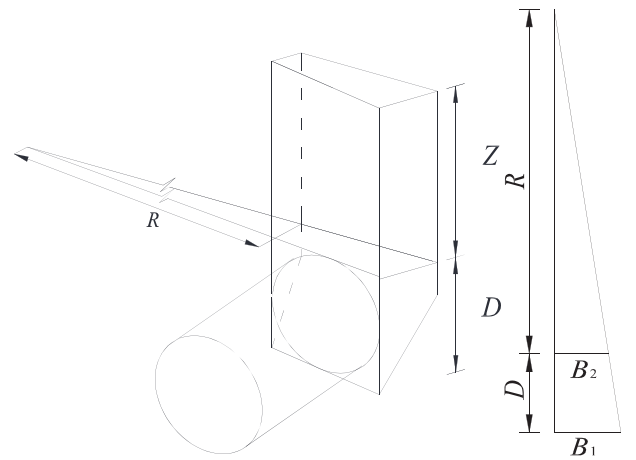

Figure 7 Connecting lines between curvature centre and shield tunnel

\section{NUMERICAL ANALYSIS OF EXCAVATION FACE STABILITY OF CURVED SHIELD 4.1 Calculation Model}

To validate the above model and its calculation method, a $100 \times 60 \times 60 \mathrm{~m}(X \times Y \times Z) 3 \mathrm{D}$ refined model 
was established on the finite difference software $\mathrm{FLAC}^{3 \mathrm{D}}$ for the curved shield tunnelling in composite strata. The model consists of 286,699 nodes in 274,944 elements. The four sides were horizontally constrained, the bottom surface was vertically constrained, and the upper boundary was simulated as a free boundary. The soil mass was simulated with Mohr-Coulomb elastoplastic model, while shield segment, grout and other concrete members were simulated as elastic materials. The calculation model is shown in Fig. 8 below.

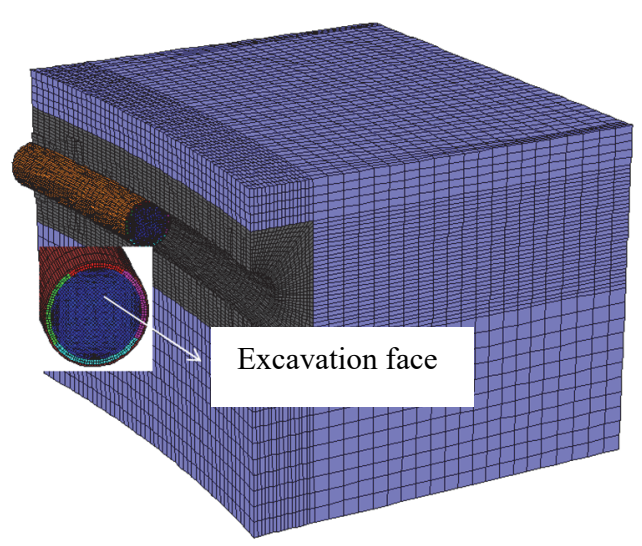

Figure 8 3D global numerical model

If the ultimate support force is defined as that measured at the sudden displacement changes of the central point in the excavation face of straight shield tunnels, there will be a huge error because of the complexity of the excavation face at the geological interface and the asymmetrical deformation of the soil mass before the excavation face under the unbalanced thrust of the curved shield tunnel in composite strata. To disclose the effects of unbalanced shield thrust on the support force of the excavation face in composite strata, the monitoring points were arranged horizontally and vertically toward the central point of the excavation face (Fig. 9).

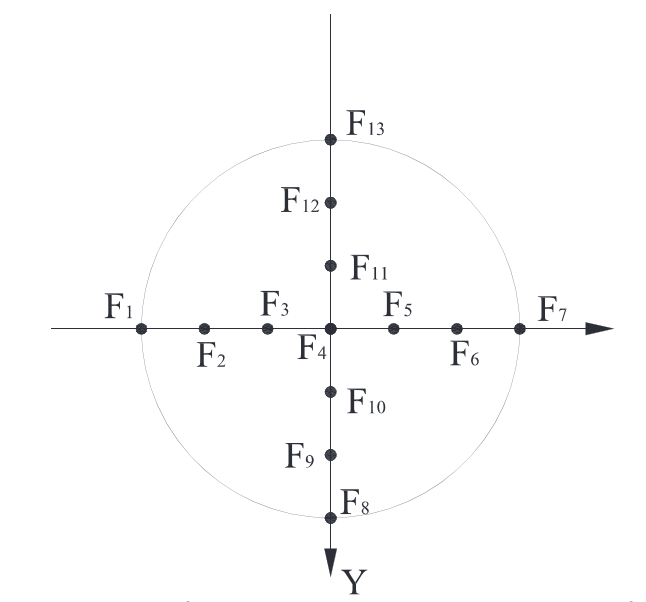

Figure 9 Layout of monitoring points on the shield excavation face

\subsection{Analysis of Numerical Results}

To visually express the relationship between the steady state, the support force, and the depth of the excavation face, the support pressure ratio $x=\sigma_{\mathrm{s}} / \sigma_{0}$ was introduced, with $\sigma_{0}$ being the lateral static earth pressure of the original stratum. Hence, the support force of the excavation face can be expressed as $\sigma_{\mathrm{S}}=x \cdot \sigma_{0}=x \cdot K \cdot \gamma \cdot Z$, where $K$ is the lateral pressure coefficient; $\gamma$ is the soil weight $(\mathrm{kPa}) ; Z$ is the tunnel depth $(\mathrm{m})$.

(1) Deformation analysis of excavation face

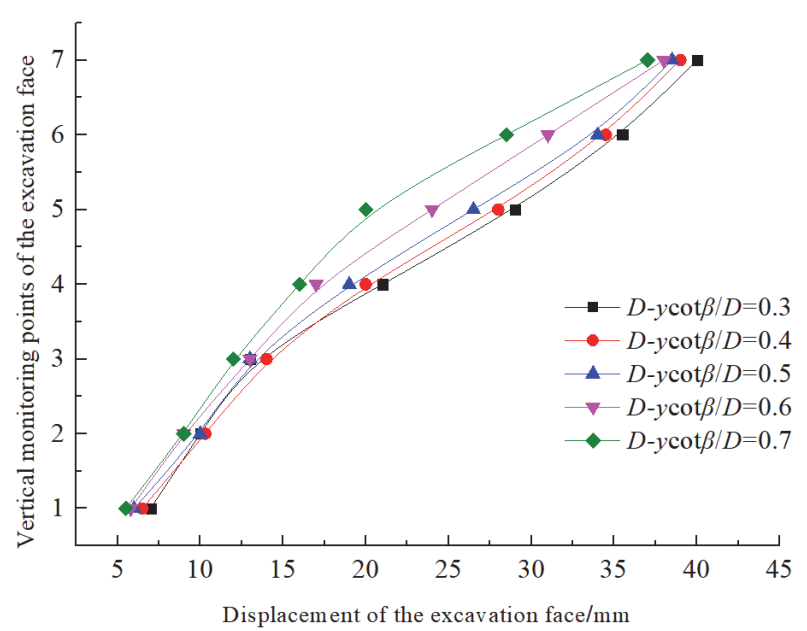

(a) Displacements of vertical diameter measuring points at different thicknesses

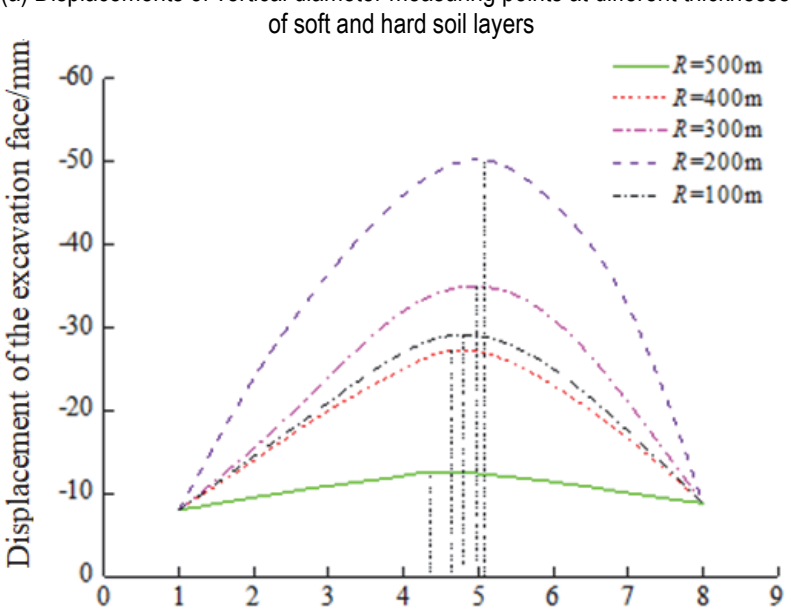

Horizontal monitoring points of the excavation face (b) Displacements of horizontal diameter measuring points at different curvature radii

Figure 10 Displacement of measuring points on the excavation face

Fig. 10a displays the horizontal displacement change of the monitoring points at the centre point of the excavation face at different thicknesses of the soft and hard layers. It can be seen that: (1) For the soil mass before the excavation face, the upper clay layer underwent a greater horizontal displacement than the lower layer of moderately weathered limestone, and maximum horizontal displacement appeared at the top of the excavation face. (2) The horizontal displacement of the excavation face decreased with the increase in the thickness of the lower layer of moderately weathered limestone; the lower part of the excavation surface was not seriously deformed thanks to the protective effect of the surrounding rock, while the upper clay layer was severely affected by disturbances; collapse accidents may occur when the maximum horizontal displacement of the excavation face approximated $40 \mathrm{~mm}$. (3) The horizontal displacement of the excavation face changed abruptly at the interface between the soft and hard layers.

Fig. 10b shows the horizontal displacement of the monitoring points at the centre point of the excavation face 
at different curvature radii. It can be seen that: (1) For the soil mass before the excavation face, the outside of the curved shield underwent a greater horizontal displacement than the inside of the curved shield, and the maximum horizontal displacement appeared on the right side of the excavation face. (2) With the increase of curvature radius, the deformation of the excavation face first increased and then decreased; the maximum displacement point of the lateral diameter initially shifted towards the right; the deviation of this point declined at the curvature radius $R=$ $100 \mathrm{~m}$; this is because the huge unbalanced thrust at the small radius damaged the surrounding rock outside the curve, while the thrust acting on the excavation face was reduced, leading to a smaller displacement at the excavation face. (3) The two sides of the excavation face had a similarly small displacement due to the protection effect of the surrounding rock.

(2) Comparison between analytical and numerical solutions

The support force measured at the sudden changes of the maximum displacement of the excavation face was adopted as the ultimate support force, and the static earth pressure was employed for calculation. Under these preconditions, the support force of the excavation face was gradually reduced until sudden changes took place to the horizontal displacement of the excavation face, indicating that the horizontal face lost stability. The support force acquired in this case is the ultimate support pressure of the excavation face.

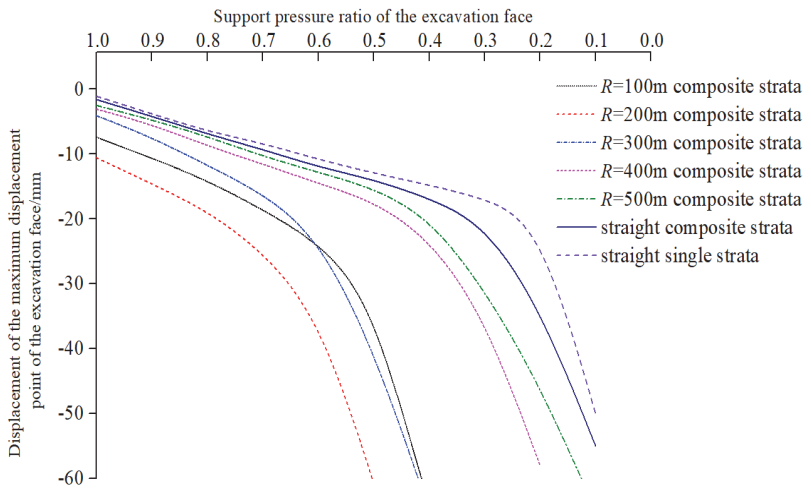

Figure 11 Relationship between ultimate support pressure ratio and excavation face displacement

Fig. 11 shows the relationships between the ultimate support pressure ratio and the displacement of the excavation face at different working conditions. The relationships were compared with the analytical solutions. According to the above-mentioned relationships between rupture angle and excavation face support force and between rupture angle and curvature radius, the author obtained indirectly the relationships between the ultimate support pressure ratio of the excavation face and radii of straight tunnels (Fig. 12).

It can be seen that: (1) The analytical solution changed in a similar trend with the numerical solution, which verifies the robustness of our calculation model; the analytical solution was slightly smaller than the numerical solution; a possible reason is as follows: During the analytical calculation, the excavated layers of the soil mass before and atop the excavation face were simplified and weighted, while the numerical calculation considered multiple layers of soil. (2) With the growth in the curvature radius, the ultimate support pressure of the curved shield tunnel in composite strata increased first and then declined, and the difference between the analytical and numerical solutions exhibited a decreasing trend; the ultimate support pressure ratio of the excavation face in the straight tunnel remained constant under the composite strata and single stratum.

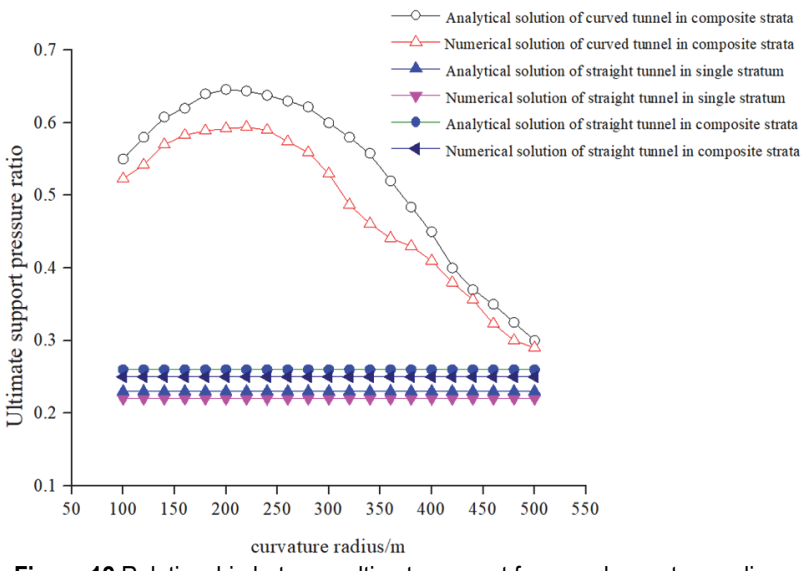

Figure 12 Relationship between ultimate support force and curvature radius

(3) Failure modes of excavation face at different radii

To analyse the soil deformation in the excavation face of shield tunnel in composite strata, the displacement nephograms were plotted for the straight shield tunnel, and the curved shield tunnel in composite strata with curvature radii of $R=300 \mathrm{~m}, R=200 \mathrm{~m}$ and $R=100 \mathrm{~m}$ (Fig. 13). It can be seen that the soil deformation of the excavation face was biased to the outside of the curve, with the decline in the curvature radius; for the straight shield tunnel, the soil deformation was distributed symmetrically along the centre of the tunnel, and the maximum deformation of the excavation face was $24.5 \mathrm{~mm}$; when the curvature radius was $R=300 \mathrm{~m}$, the soil mass before the excavation face exhibited an obvious deviation, and the maximum displacement $(31.3 \mathrm{~mm})$ appeared on the right side of the centre point of the excavation face; when the radius $R=$ $200 \mathrm{~m}$, the deformation outside the curve continued to intensify as the soil mass was subjected to the asymmetric thrust of the jack and the deformation of the shield shell, and the failure of the excavation face was transformed to the failure of the surrounding rock outside the curve; when the radius $R=100 \mathrm{~m}$, the deformation outside the curve surpassed that of the excavation face. Therefore, the curvature radius should not be too small during the actual construction.

Fig. 14 is the sectional view of the plastic zone distribution in the soil mass before the excavation face. As shown in the figure, the plastic zone before the excavation face obeyed a typical trapezoidal distribution; the soil mass surrounding the tunnel suffered from certain damages, and entered the elastic state through the redistribution of the stress; the disturbed soil mass far away from the excavation face underwent shear failure; the failure of the excavation face had a certain impact on the ground deformation, creating some plastic zones on the ground. Hence, the ultimate support pressure must be determined before shield construction. 


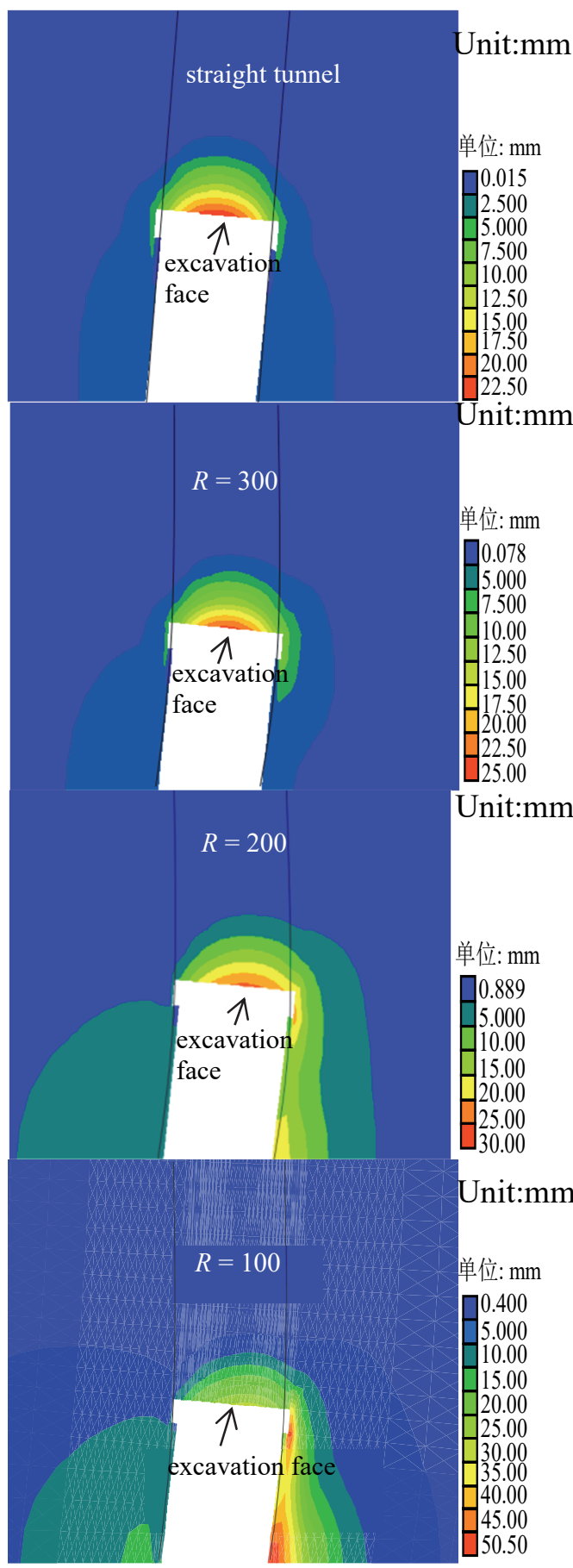

Figure 13 Soil failure modes at different radii

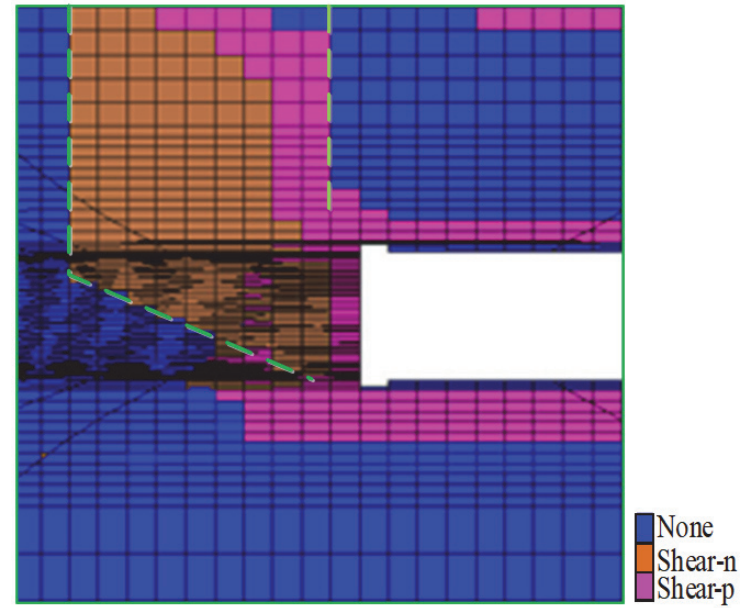

Figure 14 Distribution of plastic zones during the failure of the excavation face

\section{$5 \quad$ INFLUENCING FACTORS OF CURVED SHIELD TUNNELS IN COMPOSITE STRATA}

According to the calculation model and the support force formula, the excavation of curved shield tunnels in composite strata is under the influence of the rupture angle of the sliding surface (the rupture angle determines the thickness ratio between soft and hard layers in the composite strata), soil cohesion, and internal friction angle. The sensitivity to these factors was analysed through the proposed calculation method.

\subsection{Effect of Rupture Angle}

To disclose the effect of rupture angle on the excavation face, the rupture angle ratio between the left and right soil masses before the excavation face was defined as $\xi=\beta_{1} / \beta_{2}$. Through trial iteration, it is learned that the support force of the excavation face maximized at $\beta_{2}=$ $31.9^{\circ}$. Besides, the support force ratio acquired by increasing the value of $\beta_{1}$ without changing the value of $\beta_{2}$ was greater than that acquired by increasing the value of $\beta_{2}$ without changing the value of $\beta_{1}$. Therefore, the effect of rupture angle was examined by changing the value of $\beta_{1}$ and keeping the value of $\beta_{2}$ at $31.9^{\circ}$. Without changing the geological parameters, the value of $\xi$ was changed gradually to obtain the ultimate support pressure curve of the excavation face at different rupture angle ratios (Fig. $15)$.

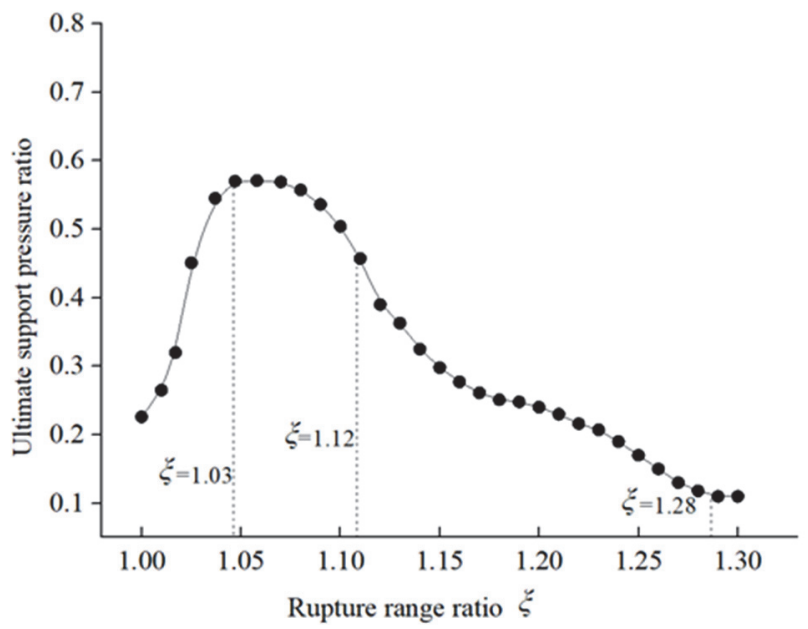

Figure 15 Ultimate support pressures of the excavation face at different rupture angle ratios

As shown in Fig. 15, the support pressure ratio was relatively small when the curvature radius of curved tunnel was large; the ultimate support pressure ratio reached the maximum at $\xi=1.03$. In this case, $\beta_{1}=32.8^{\circ}$, which is similar to the aforementioned $\beta_{1}=32.7^{\circ}$. When $\xi>1.12$, the ultimate support pressure of the excavation face plunged deeply. When $\xi>1.28$, the ultimate support force gradually stabilized.

\subsection{Effect of Cohesion}

Fig. 16 presents the ultimate support pressure ratios of curved shield tunnel at different cohesions $c$. As shown in the figure, when $\beta_{2}=31.9^{\circ}$, the ultimate support pressure 
ratio of the excavation face increased first and then decreased; with the increase of the cohesion, the support pressure ratio of the excavation face increased accordingly and peaked at $\beta_{1}=32.7^{\circ}$. The theoretical result agrees well with the simulated result, which verifies the reliability of the proposed model based on the improved silo theory. Moreover, the theoretical result is slightly below the simulated result. This is attributable to the ignorance of the groundwater seepage in theoretical calculation and the averaging treatment of the excavated soil layers.

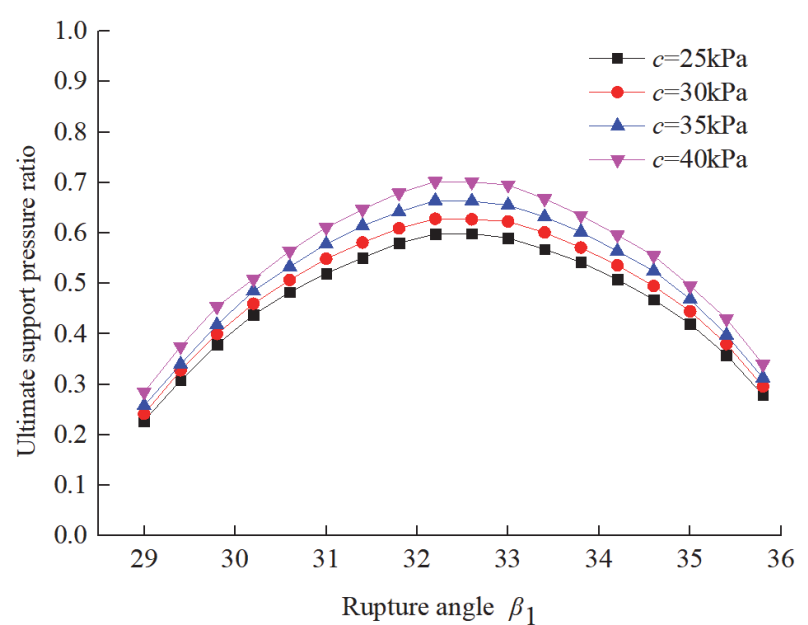

(a) Simulated result

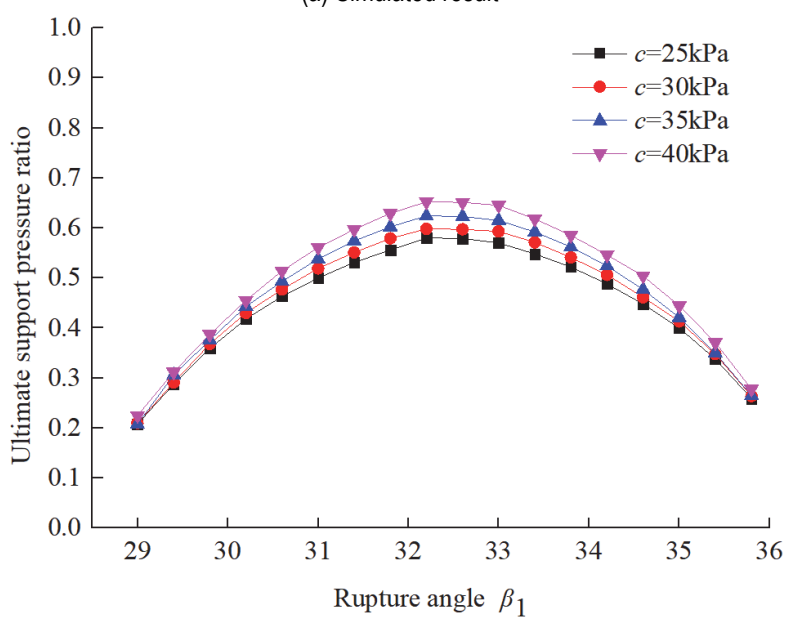

(b) Theoretical result

Figure 16 Ultimate support pressure ratios at different cohesions

\subsection{Effect of Internal Friction Angle}

Fig. 17 illustrates the ultimate support pressure ratios of the excavation face at different internal friction angles. It can be seen that the internal friction angle had a greater impact on the ultimate support pressure ratio than the cohesion; this is caused by the failure mechanism of the irregular trapezoidal sliding surfaces, in that the internal friction angle can affect the stroke of the sliding surfaces; the sliding surface of the improved silo theory model is larger than that of the conventional silo theory model; the support pressure ratio of the excavation face increased with the internal friction angle; when $\beta_{2}=31.9^{\circ}$, the ultimate support force of the excavation face increased first and then declined with the growth of $\beta_{1}$, due to the deviation of the action point of the excavation face; with the increase of $\beta_{1}$, the curvature radius shrunk, the thrust outside the curve was far greater than that inside the curve, and the action point of the excavation face continued to move; the support force of the excavation face declined when the action point moved to the edge of the excavation face.

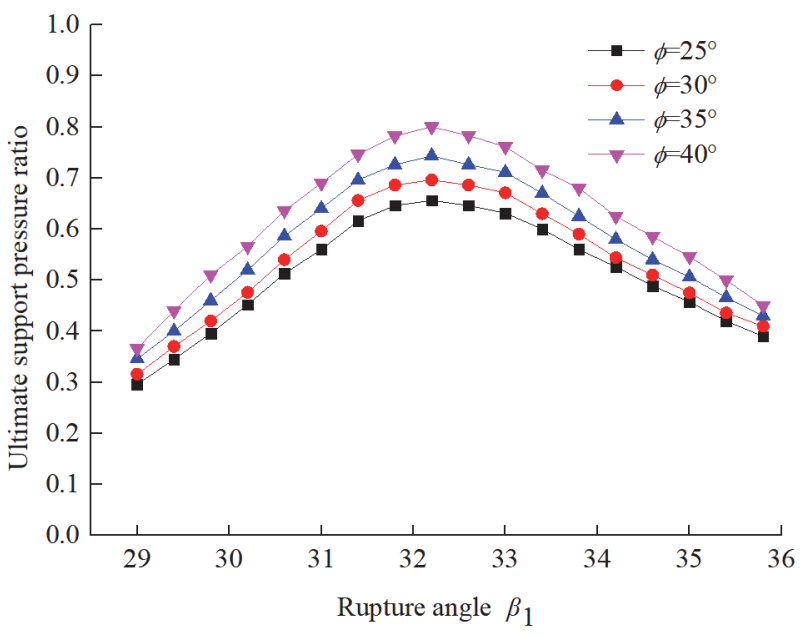

(a) Simulated result

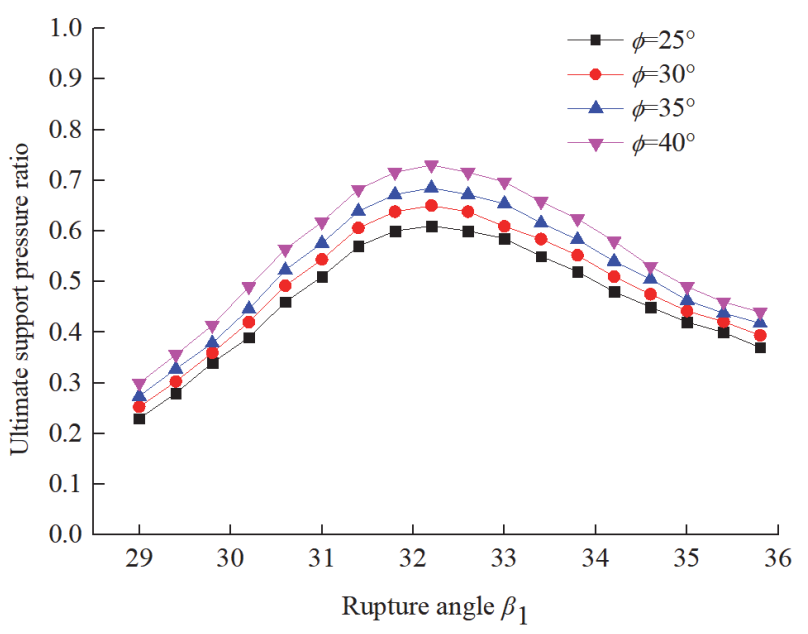

(b) Theoretical result

Figure 17 Ultimate support pressure ratios at different internal friction angles

\section{CONCLUSIONS}

(1) Unlike straight shield in single stratum, the curved shield tunnel in composite strata is featured by asymmetrical distribution of excavation face support force, sudden changes of horizontal displacement of the excavation face at the interface between hard and soft layers, and the relatively high joint force acts on the outside of the curve. These phenomena are resulted from the interface effect of the composite strata, over-excavation of the cutterhead, the extrusion of inner wall soil by shield shell, and the asymmetrical thrust of jacks.

(2) Based on the silo theory, the calculation model of the curved shield tunnel in composite strata was proposed and the calculation formula of the ultimate support force was obtained. Then, the proposed failure model was validated through a case study and comparison with numerical model.

(3) The research shows that the geometric relationship between the fracture angle of curved shield tunnel in composite strata and the curvature radius $R$ is approximately $\tan \beta_{2}=R \tan \beta_{1} /(R+D), \quad$ and the 
displacement and ultimate support pressure of the excavation face increased first and then declined with the decrease of the curvature radius.

(4) The ultimate support pressure of the excavation face in curved shield tunnel in composite strata increased with cohesion and internal friction angle, and increased first and then declined with the growth in the rupture angle difference between the left and right sides of the soil mass before the excavation face. Moreover, the internal friction angle had a greater impact on ultimate support pressure ratio than cohesion.

\section{Acknowledgements}

This work was supported by the Humanities and Social Sciences Research Foundation of the Chinese Ministry of Education (approved No. 20YJAZH022). Thank you for your support to the Humanities and Social Sciences Research Foundation of the Chinese Ministry of Education.

\section{REFERENCES}

[1] Qiao, J. L., Zhang, Y.T., \& Gao, J. (2009). Stability Analysis of Shield Tunnel Excavation Face with the Consideration of Seepage. Journal of Hydrological Engineering Geology, 31(5), 80-85.

[2] Gao, C. (2017).Research on attitude control technology of shield tunneling in soft soil stratum. Engineering Technology and Application, 3(24), 67-69.

[3] Zheng, G., Zhang, F. Z., Zhang, T., et al. (2016). Disturbance of Shield Tunnel Excavation and Compensation Grouting to Surrounding Soil: Laboratory Tests and Numerical Simulations. Chinese Journal of Geotechnical Engineering, 38(10), 1741-1753.

[4] Zhang, M. J., Wan, T., \& Li, P. F. (2018). Stability analysis of excavation face of deep-buried shield tunnel in sandy ground. Railway Construction, 58(12), 69-73.

[5] Chen, R. P., Tang, L. J., Yin, X. S., et al. (2015). An Improved 3D Wedge-prism Model for the Face Stability Analysis of the Shield Tunnel in Cohesion less Soils. Acta Geotechnica, 10(5), 683-692. https://doi.org/10.1007/s11440-014-0304-5

[6] Qin, J. S. (2005). Study on Face Deformation and Collapse of Earth Pressure Shield Tunnel. Master's thesis, Nanjing, HoHai University.

Retrieved from http://www.cnki.net/database

[7] Wang, G. F., Sun, J. C., \& Lu, L. H., et al. (2016).Study on Limit Support Pressure of Shield Tunnel Excavation Face with Mutational Geological Interface. China Rallway Sclence, 37(06), 50-59.

[8] Zhao, M. H., Mao, T., \& Niu H. Y., et al. (2016). Analysis of Limit Supporting Force of Tunnel Excavation Face for Shield Machine in Upper-hard Lower-Soft Ground. Journal of Hunan University (Natural Sciences), 43(01), 103-109.

[9] Wu, J., Liao, S. M., \& Shi, Z. H. (2015).Workface Stability of Shield Tunnel Considering Arching Effect. Journal of Tongji University(Natural Sciences), 43(02), 213-220.

[10] Yang, Y. L. (2015). Analytical Analysis of Earth Pressure Balance Shield Tunnel Face Stability and Application in Engineering. Master's thesis, Beijing: China University of Geosciences. Retrieved from http://www.cnki.net/database.

[11] Donald, I. \& Chen, Z. Y. (1997). Slope stability analysis by the upper bound approach: fundamentals and methods. Canadian Geotechnical Journal, 34(6), 853-862. https://doi.org/10.1139/t97-061

[12] Xie, J., Liu, C. G., \& Yu, H. (2006). Upper bound solutions of plastic limit analysis for the stability of two parallel circular tunnels. Journal of rock mechanics and Engineering, 25(09), 1835-1841.

[13] Terzaghi, K. (1943). Theoretical soil mechanics. New York: John Wiley and Sons Inc, 66-76. https://doi.org/10.1002/9780470172766

[14] Schmidt, B. (1996). Discussion on "Earth pressure at rest related to stress history". Canadian Geotechnical Journal, 3(4), 239-242. https://doi.org/10.1139/t66-028

[15] Varga, R. S. (1962). Matrix Iterative Analysis. Prentice-Hall. Englewood Cliffs, N. J.

[16] Young, D. M. (1971). Iterative Solution of Large Linear Systems. Academic Press New York.

[17] Zeng, W. P. (1985). On convergence of the Jacobi the GaussSeidel the SOR and the AOR iteration methods. Numerical Mathematics a Journal of Chinese Universities, 4, 5-11.

[18] Kuang, J. X. \& Ji, J. (1988). A survey of TOR methods. Journal of Computational and Applied Mathematics, 24(1), 3-12. https://doi.org/10.1016/0377-0427(88)90340-8

[19] Saady (1996). Iterative Methods for Sparse Linear. Boston PWS Pub. Co.

[20] Kelly, C. T. (1995). Iterative Methods for Linear and Nonlinear Equations. Philade-lphia U.S.A: SIAM. https://doi.org/10.1137/1.9781611970944

[21] Ike, C. C. (2018). Exponential fourier integral transform method for stress analysis of boundary load on soil Mathematical Modelling of Engineering Problems, 5(1), 3339. https://doi.org/ 10.18280/mmep.050105

[22] Dai, C. Q., Lv, Y. L., \& Hou, W. Z. (2018). Creative Teaching Model of Civil Engineering Classroom Based on Brain CognitiveScience. NeuroQuantology, 16(5), 334-340. https://doi.org/10.14704/nq.2018.16.5.1289

[23] Dai, C. Q., Zhao, Z. H., Wang, L., \& Lv, X. Z. (2018). Survey on Rheological Behaviour of WeaklyCemented Soft Rock Considering Water Deterioration. Journal of Advanced Oxidation Technologies, 21(2). https://doi.org/10.26802/jaots.2018.01824

\section{Contact information:}

Chunquan DAl, Associate Professor

College of Civil Engineering and Architecture,

Shandong University of Science and Technology,

No. 579 Qianwangang Road, Huangdao District, Qingdao, 266590, China

E-mail: dcqwin@sdust.edu.cn

Quanlei WANG, Graduate student

(Corresponding author)

College of Civil Engineering and Architecture

Shandong University of Science and Technology,

No. 579 Qianwangang Road, Huangdao District, Qingdao, 266590, China

E-mail: 1321611052@qq.com

Kun JIANG, Graduate student

College of Civil Engineering and Architecture,

Shandong University of Science and Technology,

No. 579 Qianwangang Road, Huangdao District, Qingdao, 266590, China

E-mail: 1823353034@qq.com

Tao ZHENG, Graduate student

College of Civil Engineering and Architecture

Shandong University of Science and Technology,

No. 579 Qianwangang Road, Huangdao District, Qingdao, 266590, China

E-mail: mingyue6678@126.com 